\title{
Bax/Mcl-1 balance affects neutrophil survival in intermittent hypoxia and obstructive sleep apnea: effects of p38MAPK and ERK1/2 signaling
}

\author{
Larissa Dyugovskaya ${ }^{1}$, Andrey Polyakov ${ }^{1}$, Victoria Cohen-Kaplan², Peretz Lavie ${ }^{1}$ and Lena Lavie ${ }^{1,3^{*}}$
}

\begin{abstract}
Background: Prolonged neutrophil survival is evident in various cardiovascular and respiratory morbidities, in hypoxic conditions in-vitro and in patients with obstructive sleep apnea (OSA) characterized by nightly intermittent hypoxia $(I H)$. This may lead to persistent inflammation, tissue injury and dysfunction. We therefore investigated by a translational approach the potential contribution of the intrinsic stress-induced mitochondrial pathway in extending neutrophil survival under $\mathbb{H}$ conditions. Thus, neutrophils of healthy individuals treated with $\mathbb{H}$ in-vitro and neutrophils of OSA patients undergoing nightly $\mathrm{IH}$ episodes in-vivo were investigated. Specifically, the balance between pro-apoptotic Bax and anti-apoptotic Mcl-1 protein expression, and the potential involvement of p38MAPK and ERK1/2 signaling pathways in the control of Mcl-1 expression were investigated.
\end{abstract}

Methods: Purified neutrophils were exposed to $\mathrm{H}$ and compared to normoxia and to sustained hypoxia (SH) using a BioSpherix-OxyCycler C42 system. Bax and Mcl-1 levels, and p38MAPK and ERK1/2 phosphorylation were determined by western blotting. Also, Bax/Mcl-1 expression and Bax translocation to the mitochondria were assessed by confocal microscopy in pre-apoptotic neutrophils, before the appearance of apoptotic morphology. Co-localization of Bax and mitochondria was quantified by LSM 510 CarlZeiss Microlmaging using Manders Overlap Coefficient. A paired two-tailed $t$ test, with Bonferroni correction for multiple comparisons, was used for statistical analysis.

Results: Compared to normoxia, $\mathrm{IH}$ and SH up-regulated the anti-apoptotic Mcl-1 by about 2-fold, down-regulated the pro-apoptotic Bax by $41 \%$ and 27\%, respectively, and inhibited Bax co-localization with mitochondria before visible morphological signs of apoptosis were noted. IH induced ERK1/2 and p38MAPKs phosphorylation, whereas SH induced only p38MAPK phosphorylation. Accordingly, both ERK and p38MAPK inhibitors attenuated the IH-induced Mcl-1 increase. In SH, only p38MAPK inhibition decreased Mcl-1 expression. Similar to neutrophils of healthy subjects exposed to $\mathrm{IH}(0.97 \pm 0.2)$, in OSA neutrophils, Bax/Mcl-1 ratio was significantly lower compared to normoxic controls (1.0 \pm 0.5 vs.1.99 $\pm 0.3, p=0.015)$, and Bax did not co-localize with mitochondria.

Conclusions: These findings suggest that decreased Bax/Mcl-1 balance promotes neutrophil survival in $\mathrm{H}$ in-vitro as well as in OSA patients. Moreover, Bax/Mcl-1 protein function in $\mathrm{IH}$ and $\mathrm{SH}$ might be regulated by different signal transduction pathways, highlighting a novel regulatory function through ERK1/2 signaling in $\mathrm{IH}$.

Keywords: Intermittent hypoxia, Obstructive Sleep Apnea, Neutrophil mitochondrial apoptotic pathway, Bax/Mcl-1 balance, ERK 1/2 signaling, p38MAPK signaling

\footnotetext{
* Correspondence: lenal@tx.technion.ac.il

${ }^{1}$ The Lloyd Rigler Sleep Apnea Research Laboratory, The Ruth and Bruce

Rappaport Faculty of Medicine, Technion-Israel Institute of Technology, Haifa, Israel

${ }^{3}$ Unit of Anatomy and Cell Biology, The Ruth and Bruce Rappaport Faculty of

Medicine, Technion, POB 9649, Haifa 31096, Israel

Full list of author information is available at the end of the article
} 


\section{Background}

Neutrophils are bone marrow derived short-lived cells which provide a unique model to study survival signaling. Once released into the circulation, neutrophils undergo constitutive apoptosis. However, their lifespan is prolonged in coronary syndromes such as unstable angina and acute myocardial infarction and in respiratory diseases such as chronic obstructive pulmonary disease (COPD) and neonatal and adult respiratory distress syndrome (RDS) [1-4]. Prolonged neutrophil survival is also evident in patients with obstructive sleep apnea (OSA), characterized by repeated nightly episodes of intermittent hypoxia (IH) [5]. Of note, increased neutrophil survival within tissues or in the circulation can promote persistent inflammation resulting in tissue injury and dysfunction.

Unlike in other cells, sustained hypoxia (SH) as well as IH were shown to profoundly inhibit neutrophil apoptosis in-vitro [5-9] and in-vivo [5,10]. Specifically in SH several signaling pathways and a number of family molecules that regulate apoptosis are activated. B-cell lymphocytic-leukaemia proto-oncogene $(\mathrm{Bcl})-2$ family members are one such family, which can be either proapoptotic (Bax and Bak, as well as BH3-only proteins, Bid, Bim, Bad, Bik, Noxa, PUMA) or anti-apoptotic (Mcl-1, Bcl-2, Bcl-X and A1) [11-14]. The Bcl-2 family members are integrated in cell functions at the level of the mitochondria and participate in the regulation of stress-induced apoptosis [15-17].

Bcl-2 associated X protein (Bax) is necessary for inducing apoptosis [18] and its translocation and redistribution to the mitochondria is essential for implementing the apoptotic program $[15,18,19]$. Therefore, Bax is considered a quantitative marker of early apoptotic events [13,16,20-22]. Anti-apoptotic stimuli inhibit Bax insertion into the mitochondrial membrane, thereby inhibiting its pro-apoptotic activity [13]. On the other hand, myeloid cell leukemia 1 (Mcl-1) promotes neutrophil survival by binding and sequestering Bak and Bax, which are capable of forming pores in the mitochondrial membrane [23]. Mcl-1 is up-regulated in response to various survival stimuli [23-29] and is required for neutrophil viability under $\mathrm{SH}[30,31]$. Importantly, decrease in Mcl1 levels precedes the appearance of the apoptotic morphology [32].

MAPKs, in particular p38 [33-35] and ERK [36,37] regulate the apoptotic program in neutrophils. Specifically, Mcl-1 expression might be regulated by signal transduction through ERK [28]. ERK is also responsible for increasing Mcl-1 through protein stabilization by granulocyte-macrophage colony-stimulating factor (GMCSF) [25]. Sustained hypoxia can increase neutrophil survival by activating p38MAPK signaling, thereby inducing Mcl-1 proteins [30].
Previously we have shown that NF- $\kappa B$, its downstream gene IL-8, CXCR2 receptor expression, and p38MAPK signaling pathways are essential for controlling neutrophil survival in healthy individuals treated with $\mathrm{IH}$ in-vitro via the extrinsic pathway which is Fas receptors and TNF- $\alpha$ dependent [9]. To further elucidate the mechanisms involved in prolonging neurtophil survival under IH in-vitro as well as in patients with OSA, herein we investigated the intrinsic stress-induced mitochondrial pathway. These effects of IH were investigated during the early pro-apoptotic events, which occurred in neutrophils before the appearance of morphological changes and caspase's cascade activation. Thus, we show that Bax expression was decreased and its translocation to the mitochondria was inhibited under $\mathrm{IH}$ in-vitro. Concurrently, Mcl-1 expression was up-regulated via activation of ERK1/2 and p38MAPK dependent signaling pathways. Finally, we ascertained the involvement of the mitochondrial network in prolonging the survival of neutrophils in patients with OSA. Similar to the IH in-vitro model, in OSA patients which represent an IH in-vivo model, Bax did not co-localize with the mitochondria and Bax/Mcl-1 ratio was significantly lower than in healthy controls.

\section{Methods}

\section{Neutrophil isolation and treatment}

Blood samples were obtained from 10 healthy volunteers (age $=35.8 \pm 11.9 \mathrm{yr}, \mathrm{BMI}=25.3 \pm 2.6 \mathrm{Kg} / \mathrm{m}^{2}$ ) and from 7 OSA patients $\left(\mathrm{age}=51.4 \pm 15.4 \mathrm{yr}, \mathrm{BMI}=30.2 \pm 5.5 \mathrm{Kg} / \mathrm{m}^{2}\right.$, apnea-hypopnea index $(\mathrm{AHI})=35.7 \pm 20$ events/hrs). All control subjects and OSA patients were free from cardiovascular disease or diabetes and had normal blood pressure values (not higher than 140/90 mm $\mathrm{Hg}$ ). All controls and most OSA patients did not take medications for at least 2 weeks before the study was conducted. Two OSA patients used irregularly low-dose acetyl salicylic acid (micropirin$75)$. In $7 / 10$ healthy-controls, AHI (2.1 \pm 1.8 events/hrs) was determined by a validated home monitored device (watchPAT-100 Itamar Medical, Caesarea, Israel) [38] and 3/10 controls underwent full-night polysomnography (AHI 8.0 \pm 1.7 events/hrs) as all OSA patients (Technion Sleep Medicine Center, Haifa). OSA diagnosis was based on the recommendations of the American Academy of Sleep Medicine Task Force with a cutoff point of AHI $\geq 10$ [39]. Lipid profile and high sensitivity $\mathrm{C}$-reactive protein (CRP) were determined in patients and controls as previously described [5]. The protocol was approved by the local Human Rights Committee, and all participants signed an informed consent form.

Blood samples were withdrawn under fasting conditions and polymononuclear cells (PMNs) were isolated using a two layer Ficoll-Histopaque density gradient centrifugation (Histopaque 1.077 and 1.119, Sigma-Aldrich, 
Inc., St. Louis, MO, USA). PMN purity was greater than $96 \%$, and viability was greater than $99 \%$, as determined by trypan blue exclusion. Purified PMNs were resuspended in RPMI-1640 medium, supplemented with $10 \%$ FCS and $1 \mathrm{mM}$ L-glutamine, plated without/with inhibitors and exposed to normoxia, $\mathrm{SH}$ or $\mathrm{IH}$ using the BioSpherix-OxyCycler C42 system as we described previously $[5,9]$.

\section{Light-microscopy assessment of neutrophil apoptosis}

Purified neutrophils' cytospin preparations were fixed, and stained with May Grunwald-Giemsa. Slides were read blindly by Axiovert 25 (Zeizz) light microscope. At least 300 cells/slide were analyzed. Cells showing apoptotic morphology were identified according to the following criteria: nuclear condensation in the form of a single nucleus or nuclear fragments not connected by strands $[40,41]$.

\section{In-vitro IH and SH protocol}

Purified PMNs $\left(0.6 \mathrm{ml}\right.$ per well; $3-4 \times 10^{6}$ cells $\left./ \mathrm{ml}\right)$ were plated into 24 well plates and then were exposed to normoxia, $\mathrm{SH}$ or $\mathrm{IH}$ in custom-designed incubation chambers which are attached to an external $\mathrm{O}_{2}-\mathrm{CO}_{2}-\mathrm{N}_{2}$ computer-driven controller using BioSpherix-OxyCyclerC42 system (Redfield, NY, USA). This system which enables to create periodic changes in external $\mathrm{O}_{2}$ concentrations that control air gas levels in each chamber individually was described in detail previously [5,9]. Briefly, for $\mathrm{IH}$, the $\mathrm{O}_{2}$ saturation in the medium was kept at $2 \%$ for $6.6 \pm 3.6 \mathrm{~min}$ durations, out of each $1 \mathrm{hr}$ cycle. In each experiment $6 \mathrm{IH}$ cycles were run. $\mathrm{SH}$ was employed for a comparable time at $2 \%$ actual oxygen in the medium for the entire period. Control purified PMNs were maintained in normoxic conditions for the same durations. Oxygen levels in the medium were determined by a fiber-optic dissolved oxygen electrode (BioSpherix, Redfield, NY, USA).

\section{Western blot analysis}

PMNs cultured in normoxia, IH or $\mathrm{SH}$, were lysed in Tris buffered Saline Triton -X (TBST) at $\mathrm{pH}$ 7.4 (50 mM Tris- $\mathrm{HCl}, 150 \mathrm{mM} \mathrm{NaCl}, 0.5 \%$ Triton X-100, $0.2 \mathrm{mM}$ sodium vanadate), supplemented with a mixture of protease inhibitors (Roche Diagnostics $\mathrm{GmbH}$, Roche Applied Science, Mannheim, Germany), and stored at $-80^{\circ} \mathrm{C}$ until use. Cell lysates were centrifuged at $16,000 \times g$ for $15 \mathrm{~min}$ and protein concentration was determined by Bradford reagent (Bio-Rad Laboratories $\mathrm{GmbH}$, Munich, Germany). Cell lysates $(50 \mu \mathrm{g})$ were run on $12 \%$ SDS-PAGE and transferred onto Hybond nitrocellulose membranes (Amersham Biosciences Europe GmbH, Freiburg, Germany). Membranes were blocked and incubated with primary rabbit polyclonal antibodies against Thr180/Tyr182-phosphorylated p38MAPK (Cell Signaling Technology, Inc., Beverly, MA, USA), Thr202/Tyr204-phosphorylated ERK1/2 (Cell Signaling Technology, Inc., Beverly, MA, USA), Bax (N20, sc493) and Mcl-1 (S-19, sc819, Santa Cruz Biotechnology Inc., CA, USA), followed by goat anti-rabbit IgG incubation (Amersham Biosciences Europe $\mathrm{GmbH}$, Freiburg, Germany). Then membranes were washed six times with TBST buffer and incubated with horseradish peroxidaseconjugated secondary antibody (goat anti-rabbit IgG; Amersham Biosciences Europe GmbH, Freiburg, Germany) for $1 \mathrm{hr}$ at room temperature. Densitometric analysis was performed using TotalLab TL100 v.2006c software (Nonlinear Dynamics Ltd., Newcastle Upon Tyne, UK), and is expressed in arbitrary units.

\section{Confocal laser scanning microscopy}

Viable neutrophils were stained with 100 nM MitoTracker Orange CMTMRos (Invitrogen, Molecular Probes, Eugene, Oregon, USA) for mitochondria [42]. Then fixed, Triton-permeabilized and labeled with antiBax (N20, sc493) or anti-Mcl-1(S-19, sc819) polyclonal antibodies (Santa Cruz Biotechnology Inc., CA, USA) followed by $\mathrm{Cy}^{\mathrm{TM}} 2$-conjugated Goat anti-Rabbit IgG incubation (Jackson ImmunoResearch Laboratories, Inc., Baltimore Pike, PA, USA). Nuclei were stained with TO-PRO-3 (Invitrogen Inc., Carlsbad, CA, USA). Slides were mounted with fluorescence mounting medium (Vectashield H-1000, Vector lab Inc., Burlingame, CA, USA) and were analyzed by confocal laser scanning fluorescence system (Radiance 2000) with Nikon E600 (Japan) camera. Controls for staining included a primary nonspecific rabbit IgG, secondary antibodies and fivefold excess Mcl-1 blocking peptide (sc819 P, Santa Cruz Biotechnology Inc., CA, USA).

\section{Quantitative fluorescence intensity and co-localization analysis}

Relative quantitation of green (Bax) and red (Mitochondrial staining) fluorescence of each cell was accomplished by acquiring grayscale images and fluorescence intensities were integrated using ImageJ 1.42q (Wayne Rasband National Institute of Health, USA). Co-localization of Bax and mitochondria was quantified by LSM 510 CarlZeiss MicroImaging GmbH v.4.2 R\&D in collaboration with EmBl, Heidelberg, Germany using Manders Overlap Coefficient (MOC) [43]. Only neutrophils with $\mathrm{MOC}>0.6$ were considered as cells with significant co-localization. At least 50 pre-apoptotic neutrophils from different fields were counted in each sample. 


\section{Inhibitor experiments}

MAPK inhibitors included: U0126 $(10 \mu \mathrm{M})$ for MEK1/2 blocking (Signal Transduction, Beverly, MA, USA) and SB202190 $(30 \mu \mathrm{M})$ for p38MAPK blocking (Calbiocem, EMD Chemicals, Inc., NJ, USA) $[25,44]$.

\section{Statistical analysis}

Data are expressed as mean \pm SD. A paired two-tailed $t$ test was used for single comparison of parametric data. Values of $\mathrm{p}<0.05$ were considered significant. A paired two-tailed $t$ test with Bonferroni correction was used to compare the effects of $\mathrm{IH}$ and SH vs. normoxia. Therefore, for multiple comparisons only values of $\mathrm{p}<0.017$ were considered significant. The NCSS 2004 statistical package, Kaysville, Utah, USA was used.

\section{Results}

\section{IH attenuates Bax translocation to the mitochondria and} its levels

To determine the effects of IH on neutrophil survival, apoptosis was quantified morphologically by light microscopy. The percentage of apoptotic neutrophils, as determined by a single nucleus with dense chromatin condensation, or nuclear fragments not connected by strands, was $25.0 \pm 6.3 \%$ in normoxia. Exposing neutrophils to $6 \mathrm{IH}$ cycles or to $6 \mathrm{hrs}$ of $\mathrm{SH}$ significantly decreased the percentage of apoptotic neutrophils (14.5 $\pm 6.5 \%, \mathrm{p}=0.0002 \mathrm{IH}$ vs. normoxia; $19.5 \pm 5.6 \%, \mathrm{p}=0.016$ $\mathrm{SH}$ vs. normoxia). These baseline values confirmed our earlier findings that $\mathrm{IH}$ in-vitro increased neutrophil survival $[5,9]$.

Under confocal microscopy apoptotic neutrophils were identified by the typical morphology of dense nuclei. The apoptotic neutrophils were also characterized by a very high Bax expression (green fluorescence, from 120,000 to 140,000 units), and its fusion with mitochondria (MOC $>0.6$; yellow-orange dots), as depicted in Figure 1A. Such apoptotic neutrophils, which are more prevalent in normoxia, were not investigated in further experiments, since we focused on earlier mechanisms that trigger the apoptotic program before visible signs of apoptosis can be detected. Therefore Bax expression and its translocation to the mitochondria under the three oxygen conditions were examined only in pre-apoptotic neutrophils, characterized by normal nuclear morphology (Figure 1B, C).

Neutrophils of healthy subjects were exposed to IH and compared by quantitative immunofluorescence to those exposed to $\mathrm{SH}$ and normoxia. In normoxia, preapoptotic neutrophils demonstrated intensive fusion of Bax with mitochondria, with a shift in fluorescence to yellow-orange $(\mathrm{MOC}>0.6)$, as depicted in Figure 1B. In contrast, in $\mathrm{IH}$ and $\mathrm{SH}$ treated neutrophils Bax and mitochondria were located separately with diffuse Bax distribution (green fluorescence) and the mitochondria remained dotted (red fluorescence) in the cytoplasm (Figure $1 \mathrm{C}$ ). MOC in $\mathrm{IH}$ and $\mathrm{SH}$ treated neutrophils was lower than $<0.4$, indicating that less than $40 \%$ of both components overlapped. Figure 1D summarizes the translocation of Bax and its co-localizion with mitochondria for 10 separate experiments. In normoxia, Bax translocation/co-localization (MOC $>0.6)$ was noted in $53.7 \pm 12.9 \%$ of the neutrophils. In contrast, after treatment with $\mathrm{IH}$ or $\mathrm{SH}$, the percentage of neutrophils with Bax translocating to the mitochondria was significantly decreased as compared to normoxia. However, it did not differ significantly between $\mathrm{IH}$ and $\mathrm{SH}$ treatments. Bax expression under the three oxygen conditions is summarized in Figure 1E. Since the average fluorescence intensity of Bax expression per cell varied depending on the blood donor investigated, Bax expression in normoxia in each subject was considered as $100 \%$, and the changes induced by $\mathrm{IH}$ or $\mathrm{SH}$ were plotted as a relative percentage of this value. The cumulative data (Figure 1E) show that Bax expression was significantly down-regulated in neutrophils treated by either IH (by $41 \%$ ) or SH (by 27\%), compared to normoxia. Figure $1 \mathrm{~F}-\mathrm{H}$ depicts representative confocal microscope photomicrographs of Bax expression in normoxia, $\mathrm{IH}$, and $\mathrm{SH}$. After 6 hrs of normoxia the intensity of Bax expression in pre-apoptotic neutrophils was slightly and nonsignificantly increased by $12 \%$ as compared to Time 0 .

The decrease in Bax expression in the hypoxic conditions was also confirmed by protein levels as determined by western blot analysis. The following relative values for Bax expression over $\beta$-actin were obtained: normoxia, $1.82 \pm 0.7$ units; $\mathrm{IH}, 0.96 \pm 0.2$ units ( $\mathrm{p}=0.03$ vs. normoxia) and $\mathrm{SH}, 0.97 \pm 0.5$ units ( $\mathrm{p}=0.04$ vs. normoxia). A representative immunoblot of Bax protein levels over $\beta$-actin from 6 independent experiments is depicted in Figure 2A.

\section{IH up-regulates the levels of $\mathrm{Mcl}-1$ protein}

Similar to Bax, total Mcl-1 expression was also assessed at the protein level by western blotting as illustrated in Figure 2A. The average densitometric analysis from 6 independent experiments is presented in Figure 2B. In neutrophils exposed to $\mathrm{IH}$ or to $\mathrm{SH}, \mathrm{Mcl}-1$ protein foldincrease over $\beta$-actin was significantly higher by about 2-fold compared to normoxia. Also Mcl-1 up-regulation was observed by confocal microscopy, as illustrated in Figure 2C. Similar to Bax analysis, the intensity of Mcl-1 expression in normoxia for each subject was considered as $100 \%$ and the changes induced by $\mathrm{IH}$ or $\mathrm{SH}$ were plotted as a relative percentage of this value. The specificity of Mcl-1 was confirmed using five-fold excess of the Mcl-1 blocking peptide, which abolished Mcl-1 fluorescent staining. Representative confocal microscope 


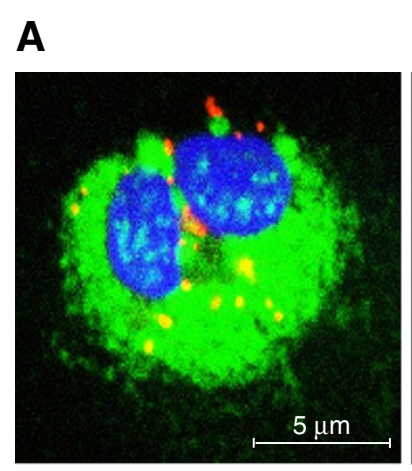

D



B



E
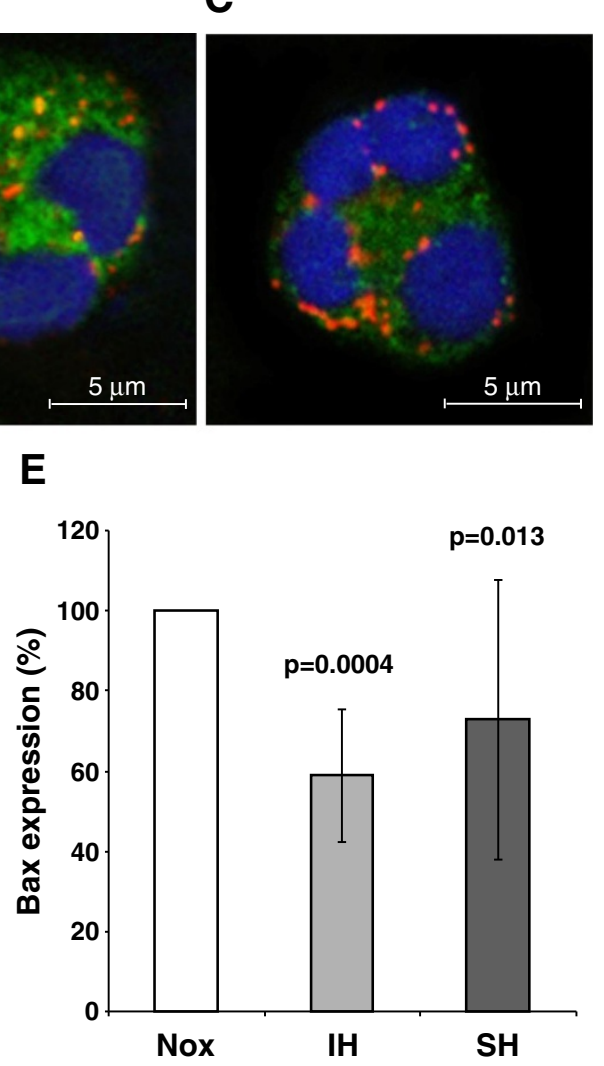

$\mathbf{F}$

G

H

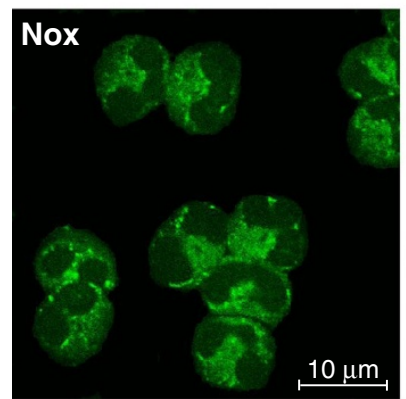

IH

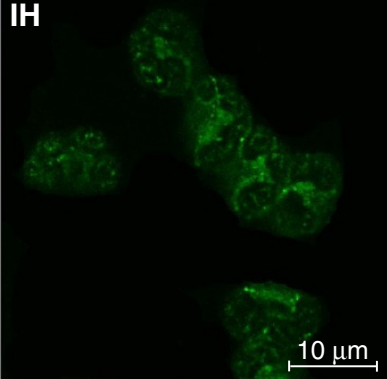

SH

Figure 1 Bax expression and co-localization with mitochondria in neutrophils exposed to various oxygen conditions. Bax expression and co-localization with mitochondria were analyzed by confocal laser scanning microscopy as described in methods. The cytoplasmic distribution of Bax (green fluorescence) and mitochondria (red fluorescence) was studied by double immunofluorescence labeling. (A) A representative photomicrograph of an apoptotic neutrophil with a very high Bax expression (140,000 arbitrary units) and intensive fusion of Bax with mitochondria (yellow), Manders Overlap Coefficient (MOC)>0.6. (B) A pre-apoptotic neutrophil with intensive Bax expression (17,000 arbitrary units). Bax is translocated and co-localized with mitochondria (yellow and orange), MOC $>0.6$ representing neutrophils predominantly seen at normoxia (Nox). (C) A pre-apoptotic neutrophil with low Bax expression (9,500 arbitrary units). Bax (green) and mitochondria (red) are located separately $(\mathrm{MOC}<0.6)$, representing neutrophils predominantly seen in intermittent hypoxia $(\mathrm{IH})$ and sustained hypoxia $(\mathrm{SH})$. (D). The percentage of

pre-apoptotic neutrophils with fusion of Bax and mitochondria (MOC>0.6) in Nox, $\mathrm{IH}$ and $\mathrm{SH}$. Values represent the means \pm standard deviations $(n=10)$. P values represent significance of $\mathrm{HH}$ or SH vs. Nox. (E) Relative percentage of Bax expression, assessed by confocal laser scanning microscopy $(n=10)$. The average fluorescence intensity unit per cell, detected by immunofluorescent quantitation at normoxia was considered as baseline (100\%), and the effects of hypoxia were calculated as relative decrease of Bax expression. P values represent significance of $\mathrm{IH}$ or SH vs. Nox. (F-H) Representative images of Bax expression (green) out of ten experiments in Nox $(\mathbf{F}), \mathbf{I H}(\mathbf{G})$, and $\mathrm{SH}(\mathbf{H})$. 
A

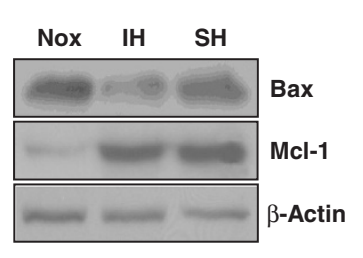

B

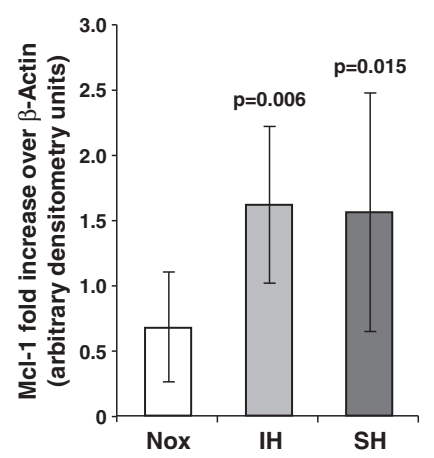

C

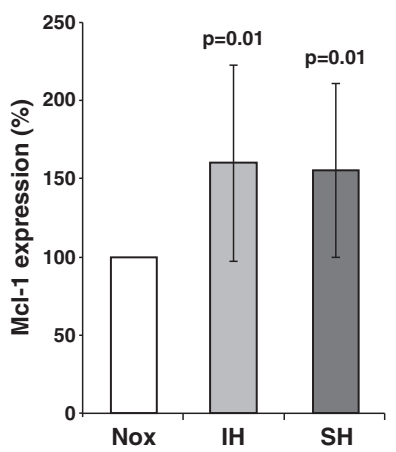

Figure $\mathbf{2}$ Mcl-1 expression in neutrophils exposed to various oxygen conditions. (A) A representative western blot depicting Bax and Mcl- 1 expression in normoxia (Nox), intermittent hypoxia $(\mathrm{IH})$ and sustained hypoxia $(\mathrm{SH})$ in one out of six experiments performed. (B) The normalized values of $\mathrm{MCl}-1$, obtained by densitometric analysis of western blots are presented as arbitrary units, and depicted as fold induction over $\beta$-actin in Nox, $\mathrm{IH}$ and $\mathrm{SH}$. (C) Mcl-1 expression was assessed by confocal laser scanning microscopy $(n=8)$. The average fluorescence intensity unit per cell, detected by immunofluorescence quantitation at normoxia was considered as baseline (100\%), and the effects of $\mathrm{IH}$ and $\mathrm{SH}$ were calculated as relative Mcl-1increase. $\mathrm{P}$ values represent significance of $\mathrm{IH}$ or SH vs. Nox.

photomicrographs of Mcl-1 expression in normoxia, $\mathrm{IH}$, and $\mathrm{SH}$ are presented in Figure 3A-C. After 6 hrs of normoxia the intensity of Mcl-1 expression in pre-apoptotic neutrophils was decreased by about $20 \%$ compared to Time 0 .

\section{Effects of ERK and p38MAPK inhibition on Mcl-1 expression}

MAPKs, including ERK1/2 and p38MAPK control neutrophil survival under certain conditions $[27,28,36,44,45]$. Specific p38MAPK and ERK1/2 inhibitors were used to determine whether Mcl-1 expression was dependent on the activation of MAPK signaling pathways under IH. Neutrophils were incubated in normoxia, $\mathrm{SH}$ or $\mathrm{IH}$ (as described in methods) with or without $10 \mu \mathrm{M}$ U0126 (a specific inhibitor of MEK1/2, blocks ERK1/2 activation) or $30 \mu \mathrm{M}$ SB202190 (a competitive p38MAPK inhibitor). Mcl-1 distribution was determined in pre-apoptotic neutrophils by confocal laser scanning microscopy. Representative images (from 1 out of 4 experiments performed) of Mcl- 1 expression in the three oxygen conditions without or with the inhibitors are presented in Figure 3A-I. Figures 3-K depict the average values of Mcl-1 expression for ERK1/2 inhibitor and p38MAPK inhibitor by relative percent when normoxia without the inhibitor was considered as $100 \%$. Blocking ERK/MEK activity slightly increased $(\mathrm{p}=0.16)$ Mcl-1 expression in normoxia but significantly decreased the IH-mediated Mcl-1 up-regulation by $40 \%$. In contrast, in $\mathrm{SH}, \mathrm{Mcl}-1$ expression was not affected by the ERK/MEK inhibitor (Figure 3D-F, J). Inhibiting p38MAPK also slightly increased $(\mathrm{p}=0.1)$ Mcl-1 expression in normoxia, but the hypoxia-induced enhanced Mcl-1 expression, was significantly attenuated in both $\mathrm{IH}$ (by $30 \%$ ) and SH (34\%) conditions (Figure 3G-I, K).
ERK and p38MAPK activation in response to hypoxia

To directly asses ERK1/2 and p38MAPK activation by $\mathrm{IH}$, their phosphorylation was determined by western blotting. As depicted in Figure 4A, only IH but not SH significantly triggered the phosphorylation of ERK1/2. This pattern of ERK1/2 activation was consistently seen in each separate experiment performed with neutrophils isolated from 6 different donors. For comparison with ERK1/2, we also confirmed our earlier findings showing that p38MAPK phosphorylation was induced in response to both $\mathrm{IH}$ and $\mathrm{SH}$ [9]. Figure $4 \mathrm{~B}$ is a representative immunoblot depicting ERK1/2 and p38MAPK phosphorylation. Non-phosphorylated controls of ERK1/ 2 and p38MAPK did not differ between the treatments.

\section{Bax expression and co-localization in neutrophils of OSA patients}

Bax expression and translocation to the mitochondria was also assessed in neutrophils of OSA patients. Neutrophils cultured for $6 \mathrm{hrs}$ in normoxia or 6 cycles of IH were compared to controls. Three out of seven studied patients were obese having a BMI $>30$. Three out of ten healthy controls were investigated concurrently with the OSA patients. All underwent full-night polysomnography after which blood samples were taken. Individual demographic, blood chemistry and sleep data for OSA patients and the controls are presented in Table 1.

The pre-apoptotic neutrophils of these control subjects expressed Bax translocation to the mitochondria under normoxia as described earlier for healthy controls (Figure 5A), and treatment with $\mathrm{IH}$ inhibited Bax/mitochondria co-localization (Figure 5B). In contrast, in patients with OSA there was little, if any, Bax translocation and co-localization to the mitochondria in normoxia (Figure 5C, E), as well as in IH (Figure 5D, F). 


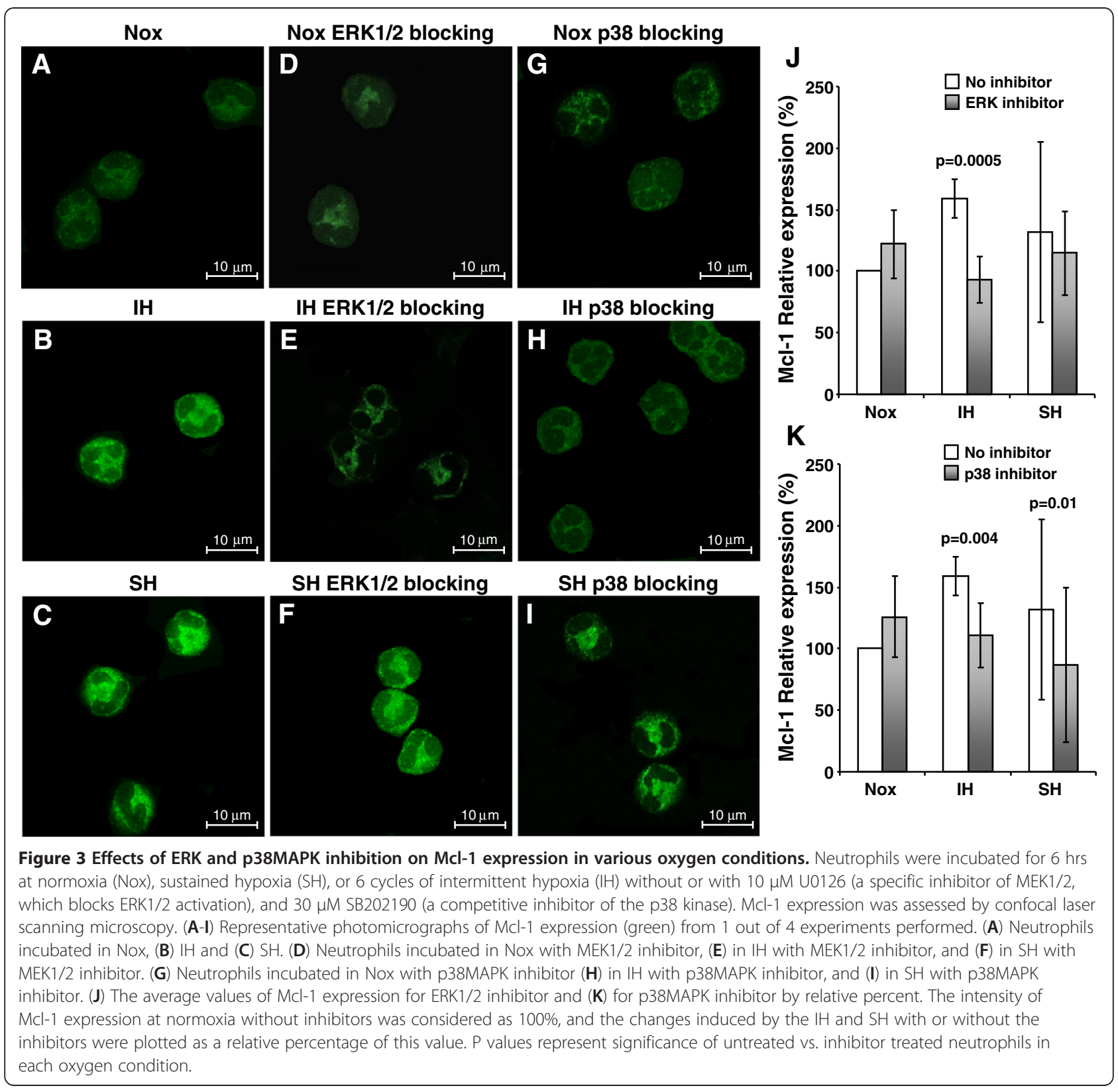

These findings were noted in non-obese patients with low CRP levels (Figure 5C, D) as well as in obese patients with high CRP levels (Figure 5E, F).

As stated above, the fluorescence intensity of Bax and Mcl-1 expression was an individual trait. We therefore used Bax/Mcl-1 ratio for comparing the redistribution of pro-/anti-apoptotic proteins between OSA and healthy controls. The average Bax/Mcl-1 ratio in normoxia was 2-fold higher in healthy controls as compared to OSA patients and was significantly decreased by about $60 \%$ and $50 \%$ after treatment with $\mathrm{IH}$ and $\mathrm{SH}$, respectively (Table 2). In OSA patients, the Bax/Mcl-1 ratio was already low at normoxia $(1.0 \pm 0.5)$ and was further decreased after exposure to IH as depicted in Table 2. Similar values were obtained for Bax/Mcl-1 ratio in normoxia immediately after harvesting the cells (data not shown).

\section{Discussion}

Neutrophils survival was shown to increase in response to IH in-vitro as well as in-vivo [5,9], however, the underlying mechanisms are not entirely understood. In the present study we investigated the contribution of the mitochondrial stress-induced pathway in prolonging neutrophil survival under $\mathrm{IH}$ treatment in-vitro and in a human IH model in-vivo. In neutrophils treated by IH 
A



B



Figure 4 ERK1/2 and p38MAPK phosphorylation in neutrophils exposed to various oxygen conditions. (A) The average ( \pm SD) normalized values of $p$-ERK1/2 $(n=6)$, obtained by densitometric analysis of western blots, are presented as fold increase over $\beta$-actin in arbitrary units in normoxia (Nox), intermittent hypoxia (IH) and sustained hypoxia (SH). (B) A representative western blot depicts the dual phosphorylated (Thr202/Tyr204) form of ERK1/2 (p-ERK1/2) and dual phosphorylated (Thr180/Tyr182) form of p38MAPK (p-p38) compared to non-phosphorylated ERK1/2 and p38MAPK in Nox, IH, and SH.

in-vitro the expression of the pro-apoptotic protein Bax was decreased, Bax translocation to the mitochondria was inhibited and the anti-apoptotic protein Mcl-1 was up-regulated via activation of ERK1/2 and p38MAPK dependent signaling pathways. In $\mathrm{SH}$ treated neutrophils, unlike in $\mathrm{IH}$, Mcl-1 up-regulation was only dependent on p38MAPK but not on ERK1/2 activation. Moreover, using a quantitative confocal microscopy analysis we have shown that the hypoxia-induced changes in Bax/Mcl-1 expression and translocation were noted in neutrophils before the appearance of apoptotic morphology. Similarly to the in-vitro findings, in OSA

Table 1 Demographic, blood chemistry and sleep apnea measures for patients and controls participating in the experiments

\begin{tabular}{|c|c|c|c|c|c|c|c|c|c|}
\hline No & Age & BMI & $\mathrm{AHI}$ & $\%$ TO Sat $<90 \%$ & $\mathrm{TG}$ (mg/dL) & Cholest. (mg/dL) & HDL (mg/dL) & $\mathrm{LDL}(\mathrm{mg} / \mathrm{dL})$ & Glucose $(\mathrm{mg} / \mathrm{dL})$ \\
\hline 1 OSA & 62 & 25.2 & 22 & 17.3 & 203.9 & 183 & 26.8 & 115.6 & 87.2 \\
\hline $2 \mathrm{OSA}$ & 64 & 27.4 & 19 & 2.2 & 50.5 & 215 & 81 & 124.3 & 88.3 \\
\hline 3 OSA & 48 & 34.2 & 33 & 6.8 & 79.9 & 154 & 60.4 & 77.5 & 95.9 \\
\hline 4 OSA\# & 74 & 35.9 & 61 & 50.2 & 116.7 & 118 & 46.3 & 48.4 & 71.3 \\
\hline $5 \mathrm{OSA}^{*} \#$ & 42 & 27.1 & 15 & 21.5 & 80.6 & 188 & 35.7 & 136.2 & 102.3 \\
\hline 6 OSA & 33 & 37.6 & 65 & 45.1 & 217.2 & 145 & 28.8 & 72.8 & 105.9 \\
\hline 7 OSA & 37 & 24.7 & 35 & 1.9 & 82.7 & 161 & 48.3 & 96 & 91.8 \\
\hline Mean & 51.4 & 30.2 & 35.7 & 20.7 & 118.8 & 166 & 46.8 & 95.8 & 91.8 \\
\hline SD & 15.4 & 5.5 & 20.0 & 19.9 & 65.7 & 32.0 & 19.2 & 31.5 & 11.4 \\
\hline 1 Cont. & 57 & 23.4 & 9 & 0 & 123.7 & 193 & 51.5 & 116.6 & 87.1 \\
\hline 2 Cont. & 38 & 25.3 & 9 & 0 & 94.2 & 185 & 59 & 107.2 & 90.7 \\
\hline 3 Cont. & 57 & 25.9 & 6 & 0 & 295.0 & 227 & 28.3 & 140.1 & 89.2 \\
\hline Mean & 50.7 & 24.9 & 8 & 0 & 171.0 & 201.7 & 46.3 & 121.3 & 89 \\
\hline SD & 11 & 1.3 & 1.7 & & 108.4 & 22.3 & 16 & 16.9 & 1.8 \\
\hline Mean $†$ & 32.9 & 25.6 & 2.1 & 0 & 107.9 & 164.2 & 39.5 & 103.1 & 86.54 .4 \\
\hline SD & 9.6 & 2.9 & 1.8 & & 60 & 16.6 & 7.4 & 18.1 & \\
\hline
\end{tabular}

$\mathrm{BMI}$ - body mass index, $\mathrm{Kg} / \mathrm{m}^{2} ; \mathrm{AHI}$ - apnea hypopnea index; $\% \mathrm{TO}_{2}<90 \%$ - Percent time asleep with arterial oxygen saturation below $90 \%$; TG - Triglycerides; Cholest.- Cholesterol; * - current smoker; \# - irregular low-dose acetyl salicylic acid usage (micropirin-75); Individual data for obstructive sleep apnea (OSA) patients and 3 control subjects (cont.) which underwent full-night polysomnography (Technion Sleep Medicine Center, Haifa). Blood samples were withdrawn under fasting after polysomnography. + Mean \pm SD of control subjects in which disordered breathing during sleep was ruled out by a validated home monitored device. 


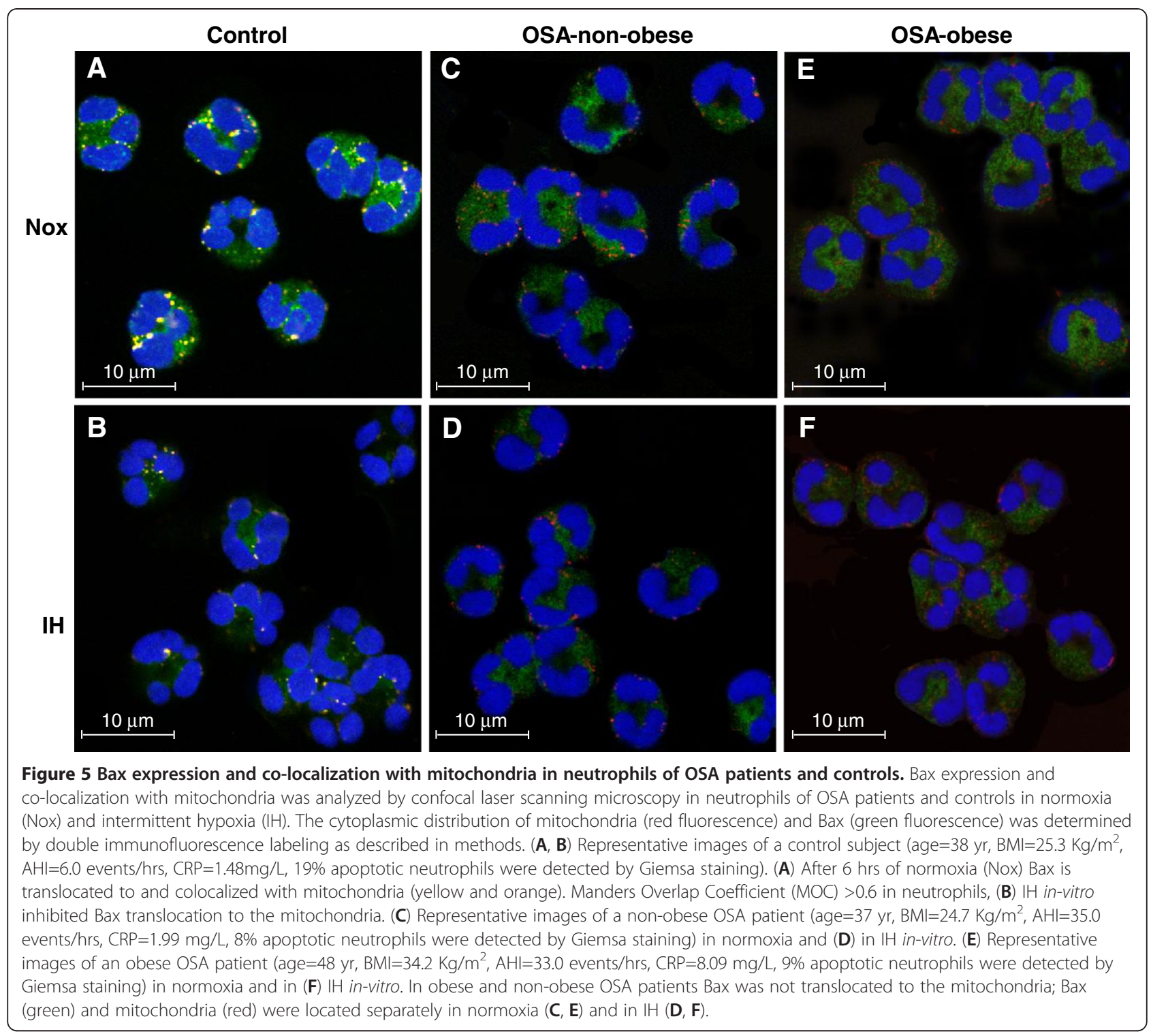

Table 2 Bax/Mcl-1 ratio in controls and OSA patients

\begin{tabular}{lccccc}
\hline $\begin{array}{l}\text { Treatment } \\
\text { groups }\end{array}$ & $\begin{array}{c}\text { Nox } \\
\text { (6 hrs) }\end{array}$ & $\begin{array}{c}\text { IH } \\
\text { (6 cycles) }\end{array}$ & $\begin{array}{c}\text { SH } \\
\text { (6 hrs) }\end{array}$ & P1 & P2 \\
\hline Controls $(n=5)$ & $1.97 \pm 0.7$ & $0.8 \pm 0.5$ & $1.05 \pm 0.6$ & $0.002 \dagger$ & 0.04 \\
\hline Controls* $^{*}(\mathrm{n}=3)$ & $2.0 \pm 0.4$ & $1.14 \pm 0.4$ & $\mathrm{ND}$ & 0.09 & \\
\hline $\begin{array}{l}\text { Controls pooled } \\
(\mathrm{n}=8)\end{array}$ & $1.99 \pm 0.3$ & $0.97 \pm 0.2$ & $\mathrm{ND}$ & 0.001 & \\
\hline OSA $(\mathrm{n}=7)$ & $1.0 \pm 0.5 \neq$ & $0.63 \pm 0.3$ & $\mathrm{ND}$ & 0.09 & \\
\hline
\end{tabular}

Normoxia (Nox), intermittent hypoxia (IH), sustained hypoxia (SH).

* - Healthy controls were investigated in parallel with OSA patients in the Sleep Lab.

ND - not determined. Values represent means \pm SD. P1 - statistical significance, IH vs. Normoxia (Nox); P2 - statistical significance, SH vs. Nox. HOnce Bonferroni correction was added to compare the effects of $\mathrm{IH}$ and $\mathrm{SH}$ vs. Nox (for multiple comparisons, values of $p<0.017$ were considered significant), only the effects of $\mathrm{IH}$ remained significant.

‡-Differences between OSA patients and controls under normoxic conditions, $\mathrm{p}=0.015$. A paired two-tailed $t$ test was used for a single comparison. patients undergoing nightly $\mathrm{IH}$, Bax did not co-localize with the mitochondria and Bax/Mcl-1 ratio was significantly lower than in healthy controls.

The Bcl-2 family of proteins is one of the key regulators of cell death at the mitochondrial level [17,19,46-49], and Bax is the best known pro-apoptotic protein. In most cell types, the expression and activity of anti-apoptotic Bcl-2 members is higher than pro-apoptotic members. By contrast, mature neutrophils constitutively express pro-apoptotic proteins and the expression of the antiapoptotic Bcl-2 members is very low $[11,50]$. Thus, the balance between pro- and anti-apoptotic members determines the fate of cells [51].

High Bax expression and its fusion with mitochondria were noted in apoptotic neutrophils by confocal microscopy analysis. Bax was also abundantly expressed, to a 
lower extent, in normoxic neutrophils of healthy subjects. However, its expression and translocation to the mitochondria were significantly lowered under $\mathrm{IH}$ as well as $\mathrm{SH}$ in-vitro. Although the critical molecules which inhibit Bax translocation to the mitochondria are as yet unknown, a possible candidate might be $\mathrm{Mcl}-1$, which was up-regulated by nearly 2 -fold under $\mathrm{IH}$ and $\mathrm{SH}$. In freshly isolated neutrophils, Mcl-1 is heterodimerized in the cytoplasm with Bax. Diminishing Mcl-1 levels release Bax from the heterocomplex Bax:Mcl-1, and allow Bax to translocate to the mitochondria where it can exercise its pro-apoptotic function [13,26,46,52]. Its translocation to the mitochondria leads to the release of pro-apoptotic factors such as cytochrome $c$, which complexes with apoptotic protease-activating factor 1 (Apaf-1) and pro-caspase- 9 to form a protein complex the 'apoptosome' which is involved in caspase-3 activation. The latter is responsible for the visible signs of apoptosis [16,19,48,51,52]. Accordingly, our findings demonstrate that changes in $\mathrm{Bax} / \mathrm{Mcl}-1$ expression and translocation to the mitochondria were noted before the appearance of apoptotic morphology, as expected, but also before caspase activation, as indicated by flow cytometry and confocal microscopy using FAN-FLICA Poly Caspase Kit (binds the activated caspases-1, -3, -4, -5, -6, $-7,-8,-9$ ) (Dyugovskaya L, Berger S, unpublished observation).

Mcl-1 has a short half-life ( $3 \mathrm{hrs})$, and spontaneous apoptosis is accompanied by Mcl-1 degradation [31]. However, its expression may be increased depending on the stimuli exerted [15]. Mcl-1 expression was increased in $\mathrm{IH}$ and $\mathrm{SH}$ in-vitro treated neutrophils compared to normoxia. It is likely that both $\mathrm{IH}$ and $\mathrm{SH}$ may induce Mcl-1 stabilization by preventing its degradation, and/or possibly by up-regulating its production. Besides in $\mathrm{IH}$ and $\mathrm{SH}$ demonstrated in this study, increased Mcl-1 levels have been previously implicated in neutrophil survival induced by LPS, cytokines such as GM-CSF, IL-1, TNF- $\alpha$, IL-15 [24-27,29], leukotriene B4 [28], Toll-likereceptor agonist [53] and $\mathrm{SH}$ for at least $8 \mathrm{hrs}$ at less than $2 \%$ oxygen [31], as obtained in this study for $6 \mathrm{hrs}$ of $\mathrm{SH}$.

Mcl-1 expression in neutrophils is regulated by a diverse array of signal transduction pathways which depend on the stimuli exerted [25,44]. Here, we demonstrate for the first time that only under $\mathrm{IH}$ the up-regulation of Mcl-1 coincided with p-ERK1/2 activation, and by inhibiting ERK1/2, the expression of Mcl-1 was inhibited. In contrast, p38MAPK was up-regulated by both $\mathrm{IH}$ as well as by $\mathrm{SH}$ as previously shown [9], and its inhibition affected $\mathrm{Mcl}-1$ expression under both hypoxic conditions. Also, like in our $\mathrm{SH}$ experimental conditions, similar findings were reported for neutrophils exposed to $12 \mathrm{hrs}$ of $\mathrm{SH}$. Inhibition of p38MAPK led to a significant decrease in Mcl-1 expression, whereas inhibiting ERK1/2 led only to a slight, but not significant decrease in Mcl-1 levels [30].

The selective ERK1/2 phosphorylation in human neutrophils by $\mathrm{IH}$ suggests that Mcl-1 activity might be regulated by different signal transduction pathways in various hypoxic conditions, such as in $\mathrm{IH}$ and $\mathrm{SH}$ demonstrated here. We should note however, that other pathways not investigated in this study, in addition to p38MAPK and ERK1/2 could be involved in the upregulation of Mcl-1 under IH. For instance, the NF-kBdependent up-regulation of IL-8 levels described earlier for $\mathrm{IH}$ [9] may control the expression of survival genes of Bcl-2 family members [54] by increasing antiapoptotic and decreasing pro-apoptotic proteins levels in neutrophils [55].

Finally we showed for the first time that in OSA patients Bax translocation to the mitochondria was minimal in neutrophils maintained at normoxic conditions, and it was further reduced in response to $\mathrm{IH}$ in-vitro in all patients investigated regardless of weight differences. Moreover, the normoxic values obtained for OSA were similar to those of control neutrophils exposed to $\mathrm{IH}$ in-vitro, illustrating the similarities between in-vitro and in-vivo IH. Additionally, the ratio Bax/Mcl-1 was significantly lower in OSA patients at normoxia as compared to control subjects clearly demonstrating that proapoptotic Bax was low whereas the anti-apoptotic Mcl-1 protein was high. Collectively, these finding suggest that the IH-dependent prolonged neutrophil survival in OSA is largely affected by the mitochondrial stress-induced pathway.

Elucidating potential mechanisms which might suppress neutrophil apoptosis by $\mathrm{IH}$ in-vivo, is of a great importance to OSA and sleep disordered breathing (SDB). OSA is a prevalent syndrome associated with cardiovascular morbidity and mortality [56]. It affects at least $4 \%$ and $2 \%$ of men and women in the adult population. However, the prevalence of SDB (IH without the characteristic excessive daytime sleepiness) is estimated to be as high as $24 \%$ and $9 \%$ in men and women. This value may rise to $60-90 \%$ in obese individuals [57]. Moreover, SDB is also highly prevalent in more than $60 \%$ of patients with acute myocardial infarction and in more than 50\% (44-93\%) of patients with stroke [56]. Furthermore, OSA and SDB are also associated with increased vascular inflammation, endothelial dysfunction and atherosclerosis [56,58].

\section{Study limitations}

The limitations of our study regarding the patients with OSA should be acknowledged. First, the IH experienced by OSA patients was not mimicked in our IH in-vitro model due to technical constrains. The short intervals as 
experienced by patients with OSA are rather difficult to reproduce with neutrophils in culture conditions because they are non-adherent cells. Therefore, the medium cannot be replaced at short intervals with alternating preconditioned hypoxic or normoxic medium. Despite that, the findings in OSA patients were similar to those obtained by IH in-vitro. Yet, it would be interesting to repeat this type of a study in an animal model of $\mathrm{IH}$, that allows mimicking the time patterns of $\mathrm{IH}$ more closely to OSA patients and in a dose dependent manner, and to avoid confounding factors and comorbidities [59].

Second, the number of OSA patients investigated in the current study is relatively small and there are significant differences in age and BMI between patients and controls. However, in a previous study [5] meticulously and rigorously investigating more than 100 patients we have shown by linear regression analysis that all apnea measures (AHI, oxygen desaturation index of 3\%, minimal oxygen desaturation, and \% time spent below 90\% saturation) were significantly correlated with decreased neutrophil apoptosis, clearly attesting to the importance of OSA severity. Moreover, using multivariate analysis we could verify that this relationship was independent of BMI, age, CRP, triglycerides, and adiponectin. Thus, although some of the patients investigated in the present study were obese and had higher CRP levels than controls, their results with respect to Bax/Mcl-1 ratio and the lack of Bax translocation to the mitochondria were identical to the results obtained in non-obese OSA patients with low CRP levels and like in the IH in-vitro, and unlike the normoxic controls' findings. It is noteworthy that in the previous study [5] we also demonstrated the contribution of the $\mathrm{IH}$ and the AHI severity measures, regardless of confounding factors, in attenuating neutrophil apoptosis by investigating OSA patients with and without treatment with nasal continues positive airway pressure (nCPAP), which ameliorates the apneas. Patients on nCPAP treatment were enrolled and were investigated on two consecutive nights, one with and one without nCPAP. Patients demographics, BMI, lipid profile or CRP were unaffected by omitting the treatment, yet all apnea severity measures were increased and neutrophil apoptosis was decreased as compared to the treatment night. These earlier data clearly emphasize the role played by the IH in increasing neutrophils lifespan, therefore allowing to investigate the mechanisms of neutrophil apoptosis under conditions of $\mathrm{IH}$ in-vivo in patients as well as in-vitro in healthy controls.

\section{Conclusions}

This study demonstrates that Bax/Mcl-1 ratio was significantly lowered in neutrophils treated by $\mathrm{IH}$ in-vitro and in patients with OSA by up-regulating the anti- apoptotic Mcl-1 and down-regulating the pro-apoptotic Bax. As a result of the IH, Bax translocation to the mitochondria was prevented. Thus, IH converts the proapoptotic phenotype into an anti-apoptotic one by modulating the Bcl-2 family members Bax and Mcl-1. This effect of IH is specifically mediated through ERK1/ 2 and p38MAPK signaling pathways whereas in $\mathrm{SH}$ it is mediated only through p38MAPK. Hence, identifying neutrophil survival pathways affected by IH may lead to new approaches in treating some sleep apnea complications associated with endothelial dysfunction and atherosclerosis. Moreover, these findings may bear relevance to other conditions and co-morbidities associated with components of IH such as physical activity, brief ascents to altitude, myocardial infarction and cancer.

\section{Abbreviations}

(OSA): Obstructive sleep apnea; (SDB): Sleep disordered breathing; $(\mathrm{IH})$ : Intermittent hypoxia; (SH): Sustained hypoxia; (GM-CSF):

Granulocyte-macrophage colony-stimulating factor; (AHI): Apnea-hypopnea index; (PMNs): Polymononuclear cells; (BCl): B-cell lymphocytic-leukaemia proto-oncogene; (Bax): BCl-2 associated X protein; (MCl-1): Myeloid cell leukemia 1, also termed myeloid cell factor-1; (MOC): Manders overlap coefficient.

\section{Competing interests}

The authors declare that they have no competing interests.

\section{Authors' contributions}

All authors have read and approved the final manuscript. The special contributions of each author are: Conception and design: LD, LL; Acquisition of data LD, LL, PL, AP, VC; Analysis and interpretation: LD, AP, PL, LL; Drafting the manuscript for important intellectual content: LL, LD AP, LP, VC; Approval of the manuscript $L L, L D A P, L P, V C$.

\section{Acknowledgments}

The authors would like to thank MS Eva Leder and the staff of the Sleep Medicine Center, Rambam Hospital, Haifa, Israel, for their invaluable help. Also, the invaluable assistance in microscopy of Dr. Edith Suss-Toby is greatly acknowledged.

This study was supported in part by a grant from the Binational Israel-US Science Foundation (Grant 2005265 to LL and PL) and partly by the Ministry of Immigration Absorption and the Committee for Planning and Budgeting of the Council for Higher Education under the framework of the KAMEA program (LD and AP).

\section{Author details}

'The Lloyd Rigler Sleep Apnea Research Laboratory, The Ruth and Bruce Rappaport Faculty of Medicine, Technion-Israel Institute of Technology, Haifa, Israel. ${ }^{2}$ Cancer and Vascular Biology Research Center, The Ruth and Bruce Rappaport Faculty of Medicine, Technion-Israel Institute of Technology, Haifa, Israel. ${ }^{3}$ Unit of Anatomy and Cell Biology, The Ruth and Bruce Rappaport Faculty of Medicine, Technion, POB 9649, Haifa 31096, Israel.

Received: 7 August 2012 Accepted: 16 October 2012 Published: 22 October 2012

\section{Reference}

1. Garlichs CD, Eskafi S, Cicha I, Schmeisser A, Walzog B, Raaz D, Stumpf C, Yilmaz A, Bremer J, Ludwig J, Daniel WG: Delay of neutrophil apoptosis in acute coronary syndromes. J Leukoc Biol 2004, 75:828-835.

2. Bianchi SM, Dockrell DH, Renshaw SA, Sabroe I, Whyte MK: Granulocyte apoptosis in the pathogenesis and resolution of lung disease. Clin Sci (Lond) 2006, 110:293-304.

3. Brown V, Elborn JS, Bradley J, Ennis M: Dysregulated apoptosis and NFkappaB expression in COPD subjects. Respir Res 2009, 10:24. 
4. Chopra M, Reuben JS, Sharma AC: Acute lung injury:apoptosis and signaling mechanisms. Exp Biol Med 2009, 234:361-371.

5. Dyugovskaya L, Polyakov A, Lavie P, Lavie L: Delayed neutrophil apoptosis in patients with sleep apnea. Am J Respir Crit Care Med 2008, 177:544-554.

6. Hannah S, Mecklenburgh K, Rahman I, Bellingan GJ, Greening A, Haslett C, Chilvers ER: Hypoxia prolongs neutrophil survival in vitro. FEBS Lett 1995, 372:233-237.

7. Walmsley SR, Print C, Farahi N, Peyssonnaux C, Johnson RS, Cramer T, Sobolewski A, Condliffe AM, Cowburn AS, Johnson N, Chilvers ER: Hypoxia-induced neutrophil survival is mediated by HIF-1alphadependent NF-kappaB activity. J Exp Med 2005, 201:105-115.

8. Mecklenburgh KI, Walmsley SR, Cowburn AS, Wiesener M, Reed BJ, Upton PD, Deighton J, Greening AP, Chilvers ER: Involvement of a ferroprotein sensor in hypoxia-mediated inhibition of neutrophil apoptosis. Blood 2002, 100:3008-3016.

9. Dyugovskaya L, Polyakov A, Ginsberg D, Lavie P, Lavie L: Molecular pathways of spontaneous and TNF-\{alpha\}-mediated neutrophil apoptosis under intermittent hypoxia. Am J Respir Cell Mol Biol 2011, 45:154-162.

10. Tamura DY, Moore EE, Partrick DA, Johnson JL, Offner PJ, Silliman CC: Acute hypoxemia in humans enhances the neutrophil inflammatory response. Shock 2002, 17:269-273.

11. Akgul C, Moulding DA, Edwards SW: Molecular control of neutrophil apoptosis. FEBS Lett 2001, 487:318-322.

12. Edwards SW, Derouet M, Howse M, Moots RJ: Regulation of neutrophil apoptosis by Mcl-1. Biochem Soc Trans 2004, 32:489-492.

13. Gardai SJ, Hildeman DA, Frankel SK, Whitlock BB, Frasch SC, Borregaard N, Marrack P, Bratton DL, Henson PM: Phosphorylation of Bax Ser184 by Akt regulates its activity and apoptosis in neutrophils. J Biol Chem 2004, 279:21085-21095

14. Witko-Sarsat V, Pederzoli-Ribeil M, Hirsch E, Sozzani S, Cassatella MA: Regulating neutrophil apoptosis: new players enter the game. Trends Immunol 2011, 32:117-124.

15. Moulding DA, Akgul C, Derouet M, White MR, Edwards SW: BCL-2 family expression in human neutrophils during delayed and accelerated apoptosis. J Leukoc Biol 2001, 70:783-792.

16. Maianski NA, Roos D, Kuijpers TW: Bid truncation, bid/bax targeting to the mitochondria, and caspase activation associated with neutrophil apoptosis are inhibited by granulocyte colony-stimulating factor. J Immunol 2004, 172:7024-7030.

17. Soriano ME, Scorrano L: The interplay between BCL-2 family proteins and mitochondrial morphology in the regulation of apoptosis. Adv Exp Med Biol 2010, 687:97-114

18. Weinmann P, Gaehtgens P, Walzog B: Bcl-XI- and Bax-alpha-mediated regulation of apoptosis of human neutrophils via caspase-3. Blood 1999, 93:3106-3115

19. Maianski NA, Maianski AN, Kuijpers TW, Roos D: Apoptosis of neutrophils. Acta Haematol 2004, 111:56-66.

20. Simon HU: Neutrophil apoptosis pathways and their modifications in inflammation. Immunol Rev 2003, 193:101-110.

21. Er E, Oliver L, Cartron PF, Juin P, Manon S, Vallette FM: Mitochondria as the target of the pro-apoptotic protein Bax. Biochim Biophys Acta 2006, 1757:1301-1311

22. van Raam BJ, Drewniak A, Groenewold V, van den Berg TK, Kuijpers TW: Granulocyte colony-stimulating factor delays neutrophil apoptosis by inhibition of calpains upstream of caspase-3. Blood 2008, 112:2046-2054

23. Thomas LW, Lam C, Edwards SW: Mcl-1; the molecular regulation of protein function. FEBS Lett 2010, 584:2981-2989.

24. Epling-Burnette PK, Zhong B, Bai F, Jiang K, Bailey RD, Garcia R, Jove R, Djeu $J Y$, Loughran TP Jr, Wei S: Cooperative regulation of $\mathrm{Mcl}-1$ by Janus kinase/stat and phosphatidylinositol 3-kinase contribute to granulocyte-macrophage colony-stimulating factor-delayed apoptosis in human neutrophils. J Immunol 2001, 166:7486-7495.

25. Derouet M, Thomas L, Cross A, Moots RJ, Edwards SW: Granulocyte macrophage colony-stimulating factor signaling and proteasome inhibition delay neutrophil apoptosis by increasing the stability of Mcl-1. J Biol Chem 2004, 279:26915-26921.

26. Moulding DA, Quayle JA, Hart CA, Edwards SW: MCl-1 expression in human neutrophils: regulation by cytokines and correlation with cell survival. Blood 1998, 92:2495-2502.
27. Pelletier M, Ratthe C, Girard D: Mechanisms involved in interleukin-15induced suppression of human neutrophil apoptosis: role of the antiapoptotic $\mathrm{Mcl}-1$ protein and several kinases including Janus kinase-2, p38 mitogen-activated protein kinase and extracellular signal-regulated kinases-1/2. FEBS Lett 2002, 532:164-170.

28. Petrin D, Turcotte S, Gilbert AK, Rola-Pleszczynski M, Stankova J: The anti-apoptotic effect of leukotriene B4 in neutrophils: a role for phosphatidylinositol 3-kinase, extracellular signal-regulated kinase and Mcl-1. Cell Signal 2006, 18:479-487.

29. Cross A, Moots RJ, Edwards SW: The dual effects of TNFalpha on neutrophil apoptosis are mediated via differential effects on expression of Mcl-1 and Bfl-1. Blood 2008, 111:878-884

30. Leuenroth SJ, Grutkoski PS, Ayala A, Simms HH: Suppression of PMN apoptosis by hypoxia is dependent on Mcl-1 and MAPK activity. Surgery 2000, 128:171-177.

31. Leuenroth SJ, Grutkoski PS, Ayala A, Simms HH: The loss of Mcl-1 expression in human polymorphonuclear leukocytes promotes apoptosis. J Leukoc Biol 2000, 68:158-166.

32. Derouet M, Thomas L, Moulding DA, Akgul C, Cross A, Moots RJ, Edwards SW: Sodium salicylate promotes neutrophil apoptosis by stimulating caspase-dependent turnover of Mcl-1. J Immunol 2006, 176:957-965.

33. Frasch SC, Nick JA, Fadok VA, Bratton DL, Worthen GS, Henson PM: p38 mitogen-activated protein kinase-dependent and -independent intracellular signal transduction pathways leading to apoptosis in human neutrophils. J Biol Chem 1998, 273:8389-8397.

34. Aoshiba K, Yasui S, Hayashi M, Tamaoki J, Nagai A: Role of p38-mitogenactivated protein kinase in spontaneous apoptosis of human neutrophils. J Immunol 1999, 162:1692-1700.

35. Alvarado-Kristensson M, Melander F, Leandersson K, Ronnstrand L, Wernstedt C, Andersson T: p38-MAPK signals survival by phosphorylation of caspase- 8 and caspase-3 in human neutrophils. J Exp Med 2004, 199:449-458.

36. Klein JB, Rane MJ, Scherzer JA, Coxon PY, Kettritz R, Mathiesen JM, Buridi A McLeish KR: Granulocyte-macrophage colony-stimulating factor delays neutrophil constitutive apoptosis through phosphoinositide 3-kinase and extracellular signal-regulated kinase pathways. J Immunol 2000, 164:4286-4291.

37. Martinez D, Vermeulen M, Trevani A, Ceballos A, Sabatte J, Gamberale R, Alvarez ME, Salamone G, Tanos T, Coso OA, Geffner J: Extracellular acidosis induces neutrophil activation by a mechanism dependent on activation of phosphatidylinositol 3-kinase/Akt and ERK pathways. J Immunol 2006, 176:1163-1171.

38. Bar A, Pillar G, Dvir I, Sheffy J, Schnall RP, Lavie P: Evaluation of a portable device based on peripheral arterial tone for unattended home sleep studies. Chest 2003, 123:695-703.

39. Sleep-related breathing disorders in adults: recommendations for syndrome definition and measurement techniques in clinical research. The Report of an American Academy of Sleep Medicine Task Force. Sleep 1999, 22:667-689.

40. Hebert MJ, Takano T, Holthofer H, Brady HR: Sequential morphologic events during apoptosis of human neutrophils. Modulation by lipoxygenase-derived eicosanoids. J Immunol 1996, 157:3105-3115.

41. Durand V, Renaudineau Y, Pers JO, Youinou P, Jamin C: Cross-linking of human FcgammaRIllb induces the production of granulocyte colony-stimulating factor and granulocyte-macrophage colony-stimulating factor by polymorphonuclear neutrophils. J Immunol 2001, 167:3996-4007

42. Maianski NA, Mul FP, van Buul JD, Roos D, Kuijpers TW: Granulocyte colony-stimulating factor inhibits the mitochondria-dependent activation of caspase-3 in neutrophils. Blood 2002, 99:672-679.

43. Manders EMM, Verbeek FJ, Aten JA: Measurement of co-localization of objects in dual-colour confocal images. J Microsc 1993, 169:375-382.

44. Saffar AS, Dragon S, Ezzati P, Shan L, Gounni AS: Phosphatidylinositol 3-kinase and p38 mitogen-activated protein kinase regulate induction of $\mathrm{Mcl}-1$ and survival in glucocorticoid-treated human neutrophils. J Allergy Clin Immunol 2008, 121:492-498. e410

45. Kilpatrick LE, Sun S, Mackie D, Baik F, Li H, Korchak HM: Regulation of TNF mediated antiapoptotic signaling in human neutrophils: role of delta-PKC and ERK1/2. J Leukoc Biol 2006, 80:1512-1521. 
46. Maianski NA, Geissler J, Srinivasula SM, Alnemri ES, Roos D, Kuijpers TW: Functional characterization of mitochondria in neutrophils: a role restricted to apoptosis. Cell Death Differ 2004, 11:143-153.

47. Fossati G, Moulding DA, Spiller DG, Moots RJ, White MR, Edwards SW: The mitochondrial network of human neutrophils: role in chemotaxis, phagocytosis, respiratory burst activation, and commitment to apoptosis. J Immunol 2003, 170:1964-1972.

48. Scheel-Toellner D, Wang KQ, Webb PR, Wong SH, Craddock R, Assi LK, Salmon M, Lord JM: Early events in spontaneous neutrophil apoptosis. Biochem Soc Trans 2004, 32:461-464.

49. Kennedy AD, DeLeo FR: Neutrophil apoptosis and the resolution of infection. Immunol Res 2009, 43:25-61.

50. Arruda MA, Barcellos-de-Souza P, Sampaio AL, Rossi AG, Graca-Souza AV, Barja-Fidalgo C: NADPH oxidase-derived ROS: key modulators of hemeinduced mitochondrial stability in human neutrophils. Exp Cell Res 2006, 312:3939-3948.

51. Cabrini M, Nahmod K, Geffner J: New insights into the mechanisms controlling neutrophil survival. Curr Opin Hematol 2010, 17:31-35.

52. Luo HR, Loison F: Constitutive neutrophil apoptosis: mechanisms and regulation. Am J Hematol 2008, 83:288-295.

53. Francois S, El Benna J, Dang PM, Pedruzzi E, Gougerot-Pocidalo MA, Elbim C: Inhibition of neutrophil apoptosis by TLR agonists in whole blood: involvement of the phosphoinositide 3-kinase/Akt and NF-kappaB signaling pathways, leading to increased levels of $\mathrm{Mcl}-1, \mathrm{~A} 1$, and phosphorylated Bad. J Immunol 2005, 174:3633-3642.

54. Cowburn AS, Deighton J, Walmsley SR, Chilvers ER: The survival effect of TNF-alpha in human neutrophils is mediated via NF-kappa B-dependent IL-8 release. Eur I Immunol 2004, 34:1733-1743.

55. Fudala R, Krupa A, Matthay MA, Allen TC, Kurdowska AK: Anti-IL-8 autoantibody:IL-8 immune complexes suppress spontaneous apoptosis of neutrophils. Am J Physiol Lung Cell Mol Physiol 2007, 293:L364-374.

56. Somers VK, White DP, Amin R, Abraham WT, Costa F, Culebras A, Daniels S, Floras JS, Hunt CE, Olson $\sqcup$, et al: Sleep apnea and cardiovascular disease: an American Heart Association/American College of Cardiology Foundation Scientific Statement from the American Heart Association Council for High Blood Pressure Research Professional Education Committee, Council on Clinical Cardiology, Stroke Council, and Council on Cardiovascular Nursing. J Am Coll Cardiol 2008, 52:686-717.

57. Malhotra A, White DP: Obstructive sleep apnoea. Lancet 2002. 360:237-245.

58. Lavie L, Lavie P: Molecular mechanisms of cardiovascular disease in OSAHS: the oxidative stress link. Eur Respir J 2009, 33:1467-1484.

59. Carreras A, Wang Y, Gozal D, Montserrat JM, Navajas D, Farre R: Non-invasive system for applying airway obstructions to mode obstructive sleep apnea in mice. Respir Physiol Neurobiol 2011, 175:164-168.

doi:10.1186/1479-5876-10-211

Cite this article as: Dyugovskaya et al:: Bax/Mcl-1 balance affects neutrophil survival in intermittent hypoxia and obstructive sleep apnea: effects of p38MAPK and ERK1/2 signaling. Journal of Translational Medicine 2012 10:211.

\section{Submit your next manuscript to BioMed Central and take full advantage of:}

- Convenient online submission

- Thorough peer review

- No space constraints or color figure charges

- Immediate publication on acceptance

- Inclusion in PubMed, CAS, Scopus and Google Scholar

- Research which is freely available for redistribution

Submit your manuscript at www.biomedcentral.com/submit
C Biomed Central 